\title{
El rastreo de cáncer de pulmón mediante tomografía computada redujo la mortalidad
}

\author{
Computed tomographic screening reduces lung cancer mortality
}

The National Lung Screening Trial Research Team. N Engl J Med 2011;365:395-409.

\section{Objetivos}

Determinar si el rastreo de cáncer de pulmón (CP) con tomografía helicoidal con baja dosis de radiación (TC-BDR), comparado contra radiografía de tórax (RxTx), reduce la mortalidad en personas de alto riesgo.

\section{Diseño}

Ensayo clínico aleatorizado.

Lugar

33 centros médicos de EE.UU.

\section{Pacientes}

53.454 pacientes de 55 a 74 años, con exposición tabáquica de al menos 30 paquetes/año y menos de 15 años de cesación (en caso de ex tabaquistas).

\section{Intervención}

TC-BDR (n: 26.722) o RxTx frente (n: 26.732) en un rastreo anual durante tres años. Se consideró caso "sospechoso" para CP a los nódulos no calcificados de al menos $4 \mathrm{~mm}$ detectados por TCBDR; o a los nódulos no calcificados, masas, derrame pleural o adenopatías detectados por RxTx. El periodo de reclutamiento fue de agosto de 2002 a abril de 2004, se realizó rastreo hasta septiembre de 2007, y el seguimiento finalizó en diciembre de 2009.

\section{Medición de resultados principales}

Mortalidad por CP y por todas las causas en ambos grupos (análisis por intención de rastrear).

\section{Resultados}

Los resultados principales se resumen en la tabla 1.

Tabla 1: Incidencia de cáncer de pulmón, y mortalidad específica y por todas las causas según método de rastreo.

\begin{tabular}{|c|c|c|c|c|c|}
\hline & \multicolumn{2}{|c|}{ Grupo TC-BDR } & \multicolumn{3}{|c|}{ Grupo $\mathrm{RxTx}$} \\
\hline & Casos & $\begin{array}{c}\text { Tasa cada } 100.000 \\
\text { personas-año (IC95\%) }\end{array}$ & Casos & $\begin{array}{c}\text { Tasa cada } 100.000 \\
\text { personas-año (IC95\%) }\end{array}$ & $\begin{array}{l}\mathrm{HR}^{\mathrm{t} / \mathrm{RRR}^{+\mathrm{t}}} \\
\text { (IC95\%) }\end{array}$ \\
\hline Incidencia de cáncer de pulmón & 1.060 & 645 & 941 & 572 & $1,13^{\dagger}(1,03$ a 1,23$)$ \\
\hline Mortalidad por cáncer de pulmón & 346 & 247 & 425 & 309 & $20 \%^{\text {t+ }}(6,8$ a 26,7$)$ \\
\hline Mortalidad por todas las causas & 1.877 & 1.080 (1.062 a 1.099$)$ & 2.000 & 1.062 (1.037 a 1.088) & $6,7 \%^{\text {+t }}(1,2$ a 13,6$)$ \\
\hline
\end{tabular}

tHR: hazard ratio. "tRRR: reducción de riesgo relativo. IC95\%: intervalo de confianza.

La tasa de adherencia al rastreo fue del 95\% para TC-BDR y del $93 \%$ para RxTx. Entre los resultados positivos, $96,4 \%$ en el grupo TC-BDR y $94,5 \%$ en el grupo RxTx fueron falsos positivos. La tasa de al menos un efecto adverso luego del rastreo fue de $1,4 \%$ en el grupo TC-BDR y $1,6 \%$ en el RxTx. En el grupo TC-BDR se calculó un número necesario a rastrear (NNR) de 320 (para prevenir una muerte por CP).

\section{Conclusiones}

El rastreo utilizando TC-BDR reduce la mortalidad por cáncer de pulmón.

Fuente de financiamiento: Cancer Imaging Program, Division of Cancer Treatment and Diagnosis; y Early Detection Research Group and Biometry Research Group, Division of Cancer Prevention.

\section{Comentario}

El cáncer de pulmón es la primera causa de muerte por cáncer para ambos sexos en los EE.UU. ${ }^{1}$ y para hombres en la Argentina ${ }^{2}$. Esto justifica la búsqueda de métodos de rastreo que ayuden al diagnóstico precoz, y así, a su tratamiento. Sin embargo, hay que señalar que este ensayo compara dos método de diagnóstico que no constituyen un estándar de la práctica habitual, siendo de hecho la recomendación de la fuerza de tareas preventiva norteamericana (USPSTF) respecto al rastreo de $\mathrm{CP}$, de tipo I (evidencia insuficiente para expedirse a favor o en contra) ${ }^{3}$.

Se plantean como limitaciones del presente trabajo: la frecuencia elevada de resultados falsos positivos; la detección de neoplasias que tal vez nunca hubiesen sido sintomáticas (sobrediagnóstico); la conducción del estudio en instituciones reconocidas por su experiencia en radiología, diagnóstico y tratamiento de cáncer; el riesgo potencial de desarrollo futuro de cáncer inducido por radiación ionizante; y la incertidumbre respecto de la frecuencia y ventana óptimas del rastreo.
La reducción de la mortalidad secundaria a la implementación de una determinada tecnología diagnóstica es relevante, sin embargo no constituye la única variable necesaria para conducir a la implementación de dicha tecnología como política de salud. Merecen también consideración aspectos como la costo-efectividad, la accesibilidad de la técnica, y el balance entre beneficios y daños potenciales.

\section{Conclusiones del comentador}

Por el momento este resultado no cambiará nuestra práctica habitual (no rastreo del CP) y habrá que esperar la síntesis de mayor evidencia.

Conociendo tan claramente que el tabaco es el principal agente causal de esta enfermedad y que evitar su consumo crónico es la mejor forma de prevenirla, como médicos de cabecera nuestra mejor intervención es el consejo antitabáquico, identificar a los fumadores y brindarles nuestra ayuda para que tengan éxito para abandonarlo.

María de las Nieves Ganiele [ Servicio de Medicina Familiar y Comunitaria del Hospital Italiano de Buenos Aires. maria.ganiele@hospitalitaliano.org.ar ]

Ganiele, MN. El rastreo de cáncer de pulmón mediante tomografía computada redujo la mortalidad. Evid Act Pract Ambul. Ene-Mar 2012;15(1):9. Comentado de: The National Lung Screening Trial Research Team. Reduced lung-cancer mortality with low-dose computed tomographic screening. N Engl J Med 2011;365:395-409. PMID: 21714641.

\section{Referencias bibliográficas}

1. U.S. Cancer Statistics Working Group. United States Cancer Statistics: 1999-2007 Incidence and Mortality Web-based Report. Atlanta: U.S. Department of Health and Human Services, Centers for Disease Control and Prevention and National Cancer Institute; 2010.

2."Mortalidad por tumores malignos, 1993-1996 y 1997-2000". Boletín Nro 96. Programa Nacional de Estadísticas de Salud. Dirección de Estadísticas en Informaciones de Salud, Ministerio de Salud, República Argentina. Buenos Aires, junio de 2002.

3.U.S. Preventive Services Task Force. Lung cancer screening (en línea). AHRQ Pub. No. 04-0537-A. 2004. Disponible en URL: http://www.uspreventiveservicestaskforce.org/3rduspstf/lungcancer/lungcanrs.pdf 